\title{
Photovoltaic Technique for Measuring Resistivity Variations of High Resistivity Silicon Slices*
}

\author{
D. L. Blackburn
}

Institute for Applied Technology, National Bureau of Standards, Washington, D.C. 20234

January 13, 1978

\begin{abstract}
A description of an automated, photovoltaic system for measuring the resistivity variation of high resistivity, large diameter silicon wafers is given. The photovoltaic technique utilizes a scanning light spot to induce a bulk photovoltage and a change in resistance from which is calculated the local variation in resistivity. This nondestructive technique requires no contacts to the useful fabrication area of the wafer, and measured results have good correlation with the results of the four-probe technique. Specific examples of measured resistivity gradients are presented along with a discussion of the theory, measurement conditions and limitations, and description of a calculator-based automated system to perform the measurements.
\end{abstract}

Key Words: Automation; computer control; homogeneity; measurement method; photovoltage, bulk; power semiconductor materials; resistivity; semiconductors; silicon; thyristors; transistors, power.

\section{Introduction}

The radial variation of resistivity is one of the principal characteristics specified in the procurement of silicon for the manufacture of high power semiconductor devices. Resistivity variations result from an inhomogeneous doping density in the starting silicon and both the solid state diffusion and alloying steps used in the fabrication of high power devices are affected by such inhomogeneities.

Gross variations in resistivity can cause wide variations in device characteristics and contribute to poor device yields. Poor junction geometry and nonuniform current distributions are frequent problems in devices fabricated from inhomogeneous material. Failures due to hot spots or thermal runaway may result. Also, localized low resistivity regions of a wafer limit operating voltage of a device fabricated from that wafer to values lower than those expected from the remainder of the wafer.

The method commonly used for determining the resistivity variations of starting silicon wafers is the four-probe technique. There are a number of difficulties and limitations with this method. A basic limitation in the application of this method to the measurement of resistivity variations is the relatively large sampling region of the probe $[1] .{ }^{1}$ Because of this, it has been recommended that to obtain better spatial resolution, a two-probe measurement be made along a narrow bar cut from along the measurement diameter of interest [2]. There can also be large errors introduced into the four-probe measurement due to slight mislocations of the probes, particularly near the wafer edge $[1,3]$. The spreading resistance technique has none of these difficulties, but it can be very slow and time consuming. Perhaps the greatest limitation of any probing method such as spreading resistance or four-probe is the placement of probes onto the wafer surface in precisely those areas where diffusions and other

* This work was conducted as part of the NBS program on Semiconductor Measuremen Technology and was funded principally by the Energy Research and Development Administration, Divisions of Administration and Electrical Energy Systems, trhough ERDA Task Order A021-EES.

${ }^{1}$ Figures in brackets indicate literature references at the end of this paper. processing steps are to occur. The damage caused by the probes may itself be detrimental to the reliability and yield of devices fabricated in these slices.

The photovoltaic technique offers an alternative for measuring resistivity variations along the diameter of circular wafers. The technique, which is based upon the theory of Tauc [4], requires no contact with the wafer surface in the area where a finished device or devices are to be fabricated. The validity of a modified photovoltaic theory for circular wafers was demonstrated several years ago [5]. The present work has established the feasibility of automating the photovoltaic technique for the rapid measurement of radial resistivity variations in high-resistivity, large-diameter, power grade silicon.

\section{Theory of the Photovoltaic Effect}

The underlying theory of the bulk photovoltaic effect was derived and its physical aspects discussed by Tauc [4]. The bulk photovoltaic effect is a phenomenon which occurs when electron-hole pairs are photogenerated in a region of an impurity-density gradient (or, equivalently, a resistivity gradient). In such a region, an internal electric field exists in the semiconductor. This is much like the situation at a $p n$ junction, except that the field is much smaller than for a junction. When excess electron-hole pairs are generated in this region, they are separated by the internal electric field, and the steady-state distribution of the separated carriers is such that the magnitude of the net internal field is reduced. It is the reduction in magitude of the internal field which results in the photovoltage which can be measured at contacts made to the specimen. It is possible to relate this measured photovoltage to the resistivity gradient in the region where the excess electron-hole pairs are distributed.

The physics of the photovoltaic effect in one dimension is described graphically in figure 1 . Figure la is a representation of a typical radial resisitivity profile found in highresistivity Czochralski-grown silicon. Figure $1 \mathrm{~b}$ shows the band structure for such a resistivity variation and illustrates 


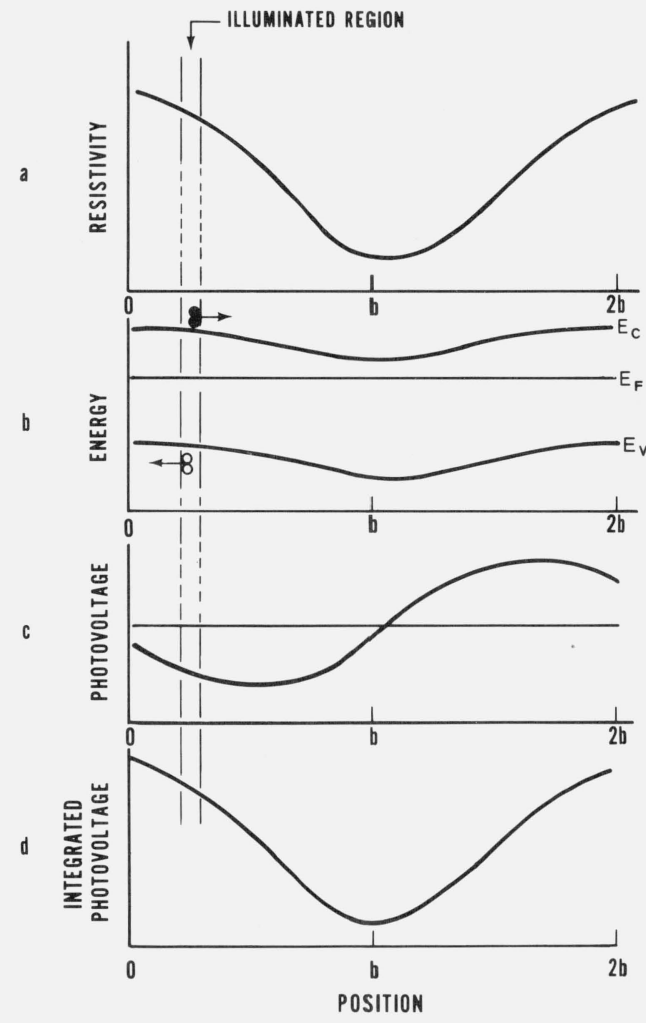

FIGURE 1. A representation of (a) a typical resistivity profile, (b) the associated energy band structure, (c) photovoltage, and (d) integrated photovoltage for an ntype specimen.

how the internal field (as manifested by the variation with position of the conduction and valence band edges) causes the photogenerated electron-hole pairs to be physically separated. Thus, a local voltage dipole is created. This results in a voltage measurable at the ends of the specimen. If the light is scanned along the specimen, a voltage profile can be determined, as shown in figure lc. If this voltage is integrated over position, assuming that the steady-state excess density of photogenerated carriers is independent of position, and then multiplied by a constant dependent only upon known material parameters, one obtains the profile in figure $1 \mathrm{~d}$. This profile is identical to the variation in resistivity, as shown in figure la. Because the entire profile in figure $1 \mathrm{~d}$ is shifted by an unknown integration constant, this method determines the variation in resistivity and not the absolute resistivity at each position. It will be shown later that a measurement of a weighted average specimen resistivity using the van der Pauw technique [6] can be employed to estimate the absolute resistivity at each position from the photovoltaic resistivity variation profile.

For most specimens, the excess density of steady-state photogenerated carriers is not constant with position. This is principally because the effective lifetime of the material is not constant but varies with position. This effect is accounted for in this measurement technique by also measuring the photoinduced change in specimen resistance as a function of position.
For circular specimens, where a small light spot falls on a wafer diameter and the photovoltage is measured at the ends of that diameter, the measured voltage also depends upon the position of the light spot on the diameter. The equation relating the resistivity variation to the photovoltage and photoinduced change in specimen resistance for a circular semiconductor wafer is:

$$
\frac{d \rho}{d x}=\frac{q}{\pi k T} \frac{\left(1+\frac{\mu_{M}}{\mu_{m}}\right)}{1-(x / b)^{2}} \frac{\bar{\rho}^{2}}{b t} \frac{V(x)}{\Delta R(x)}
$$

where $b$ and $x$ are shown in figure 2 and

$$
\begin{array}{ll}
\frac{d \rho}{d x} & =\text { resistivity gradient }(\Omega \cdot \mathrm{cm} / \mathrm{cm}) \\
q & =\text { majority carrier charge }(\mathrm{C}) \\
k & =\text { Boltzmann's Constant }(\mathrm{J} / \mathrm{K}) \\
T & =\text { temperature }(\mathrm{K}) \\
\mu_{M} & =\text { majority carrier mobility }\left(\mathrm{cm}^{2} / \mathrm{V} \cdot \mathrm{s}\right) \\
\mu_{m} & =\text { minority carrier mobility }\left(\mathrm{cm}^{2} / \mathrm{V} \cdot \mathrm{s}\right) \\
\bar{\rho} & =\text { average resistivity }(\Omega \cdot \mathrm{cm}) \\
t & =\text { thickness }(\mathrm{cm}) \\
V(x) & =\text { photovoltage }(\mathrm{V}) \\
\Delta R(x) & =\text { photoinduced change in resistance }(\Omega)
\end{array}
$$

Equation (1) differs from the equation for $d \rho / d x$ derived previously [5] by a multiplicative factor of $4 / 3$. In the course of this work, a more exact analysis of the effect of the circular geometry upon the measured photovoltage and photoinduced change in specimen resistance has shown that eq (1) is a better approximation for $d \rho / d x$ than that presented previously.

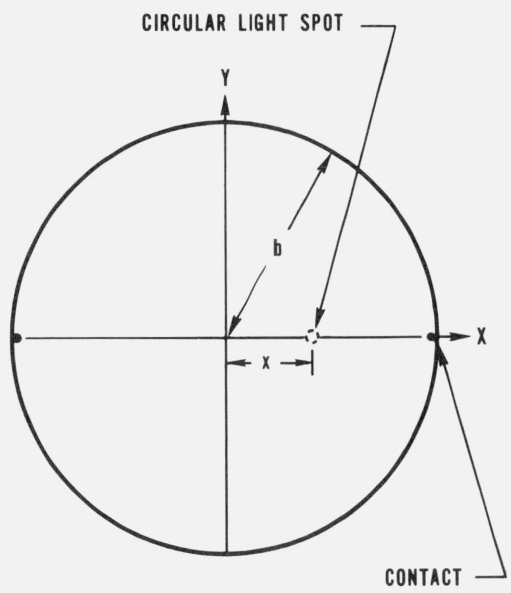

FigurE 2. The wafer geometry upon which eq (1) is based.

\section{Measurement Procedure}

To measure the resistivity gradient using the photovoltaic technique, the circuit shown in simplified form in figure 3 is used. First, the photovoltage, $V(x)$, is measured as a function 


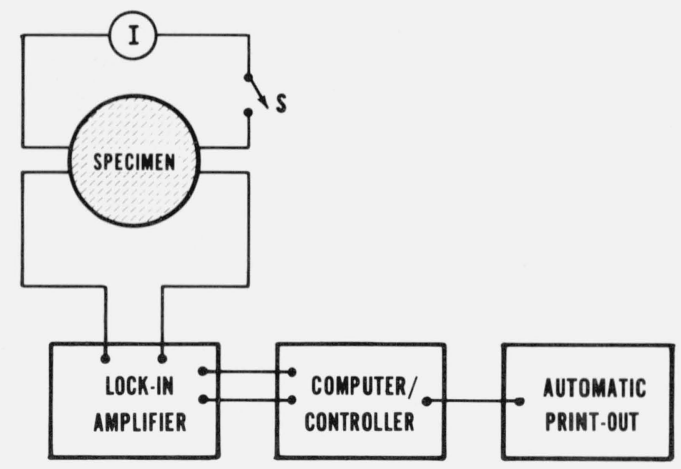

FigURE 3. Simplified version of the measurement system.
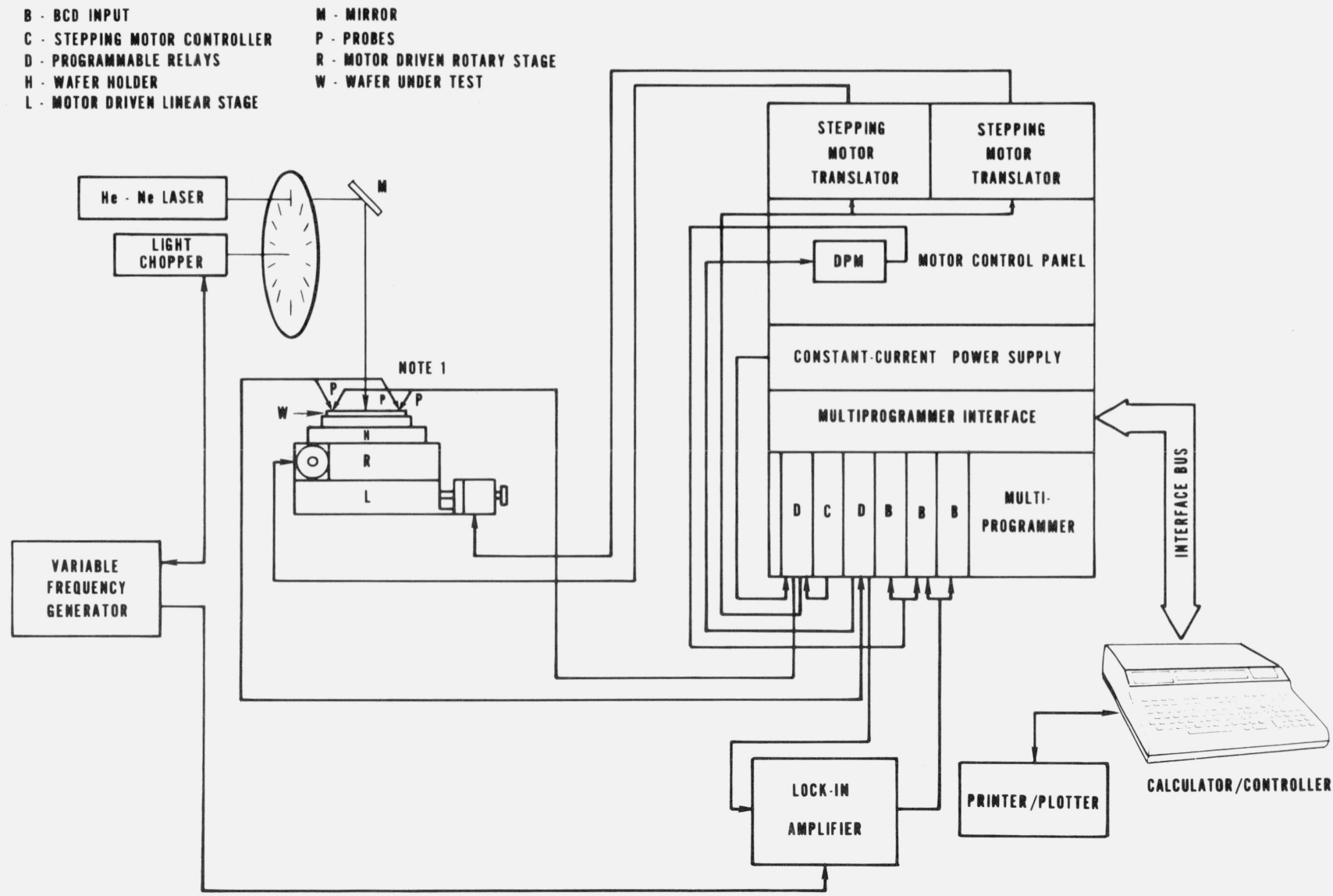

NOTE 1: THE PROBES ARE ACTUALLY PERPEMDiCULAR tO THE SURFACE OF the WAFER UNOER teSt.

FigURE 4. Schematic of automated measurement system.

of position. This is done by having the switch $\mathrm{S}$ open, stepping the wafer beneath the light probe in constant increments, $\Delta x$, and measuring $V(x)$ at each position.

Next, the photoinduced change in specimen resistance, $\Delta R(x)$, is measured. This is accomplished by closing switch $\mathrm{S}$ and again stepping the wafer beneath the light probes in increments of $\Delta x$ such that each $\Delta R(x)$ is measured at the same positions as $\mathrm{V}(x)$. Because a constant current, $I$, exists in the specimen with the switch closed, a decrease in the voltage across the specimen occurs as the light sweeps along the diameter. This is because the total specimen resistance is decreased due to the localized decrease in resistivity caused by the excess electron-hole pairs generated by the light. If the change in voltage is $\Delta V(x)$, then: 


$$
\Delta R(x)=\frac{\Delta V(x)}{I} .
$$

The quantities $V(x)$ and $\Delta R(x)$ are then inserted in eq (1) to compute the gradient, $d \rho / d x$, at each position, $x$. To determine the variation in $\rho$, the plot of $d \rho / d x$ is numerically summed $\left(\rho_{m}(x)=\sum_{i=1}^{m}(d \rho / d x)_{\mathrm{i}} \Delta x\right)$. Thus, a plot of the variation in resistivity is obtained. As mentioned, an absolute determination of resistivity is not obtained, but each $\rho_{m}(x)$ above differs from the absolute value by a constant, $\rho_{0}$, the value of the resistivity at the position the summation was begun. To estimate the value of $\rho_{0}$, the average of all the $\rho_{m}(x)$ 's $\left(\rho_{\mathrm{mAVG}}=1 / N \sum_{m=1}^{N} \rho_{\mathrm{m}}(x)\right)$ is subtracted from the measured van der Pauw average resistivity, $\bar{\rho}[6]$. This value for $\rho_{0}$ is then added to each $\rho_{m}(x)$ to determine the absolute resistivity profile. The van der Pauw average resistivity measurement has been automated and is an intrinsic part of the measurement system.

\section{Apparatus}

A schematic of the automated photovoltaic measurement system is shown in figure 4 . The entire measurement system is under the control of a computer/controller. The results of the measurements are furnished in hard copy by the printer/ plotter.

He Ne Laser-A laser, operating at $0.6328-\mu \mathrm{m}$ wavelength, is used as the source for generating the electron-hole pairs in the silicon. The laser used in this work has about a $15-\mathrm{mW}$ power output at $0.6328 \mu \mathrm{m}$. The laser can also be operated at $1.152 \mu \mathrm{m}$ with $>2 \mathrm{~mW}$ of power output. It has been found, however, that the internal reflections within the silicon at this longer, very penetrating, wavelength can induce a barrier photovoltage at the measurement contacts which masks the bulk photovoltage generated at the light spot position. The $0.638-\mu \mathrm{m}$ radiation is all absorbed very near the silicon surface and so is not reflected internally to the contact region. The laser is used because of the high energy density easily obtained within a small spot about 1 $\mathrm{mm}$ in diameter.

Lock-in Amplifier - The magnitude of the photovoltage is usually very small. In this work, for specimens with resistivity between 50 and $200 \Omega \cdot \mathrm{cm}$, the voltage is of the order of $\pm 1 \mu \mathrm{V}$. For specimens with resistivity between 10 and 50 $\Omega \cdot \mathrm{cm}$, the photovoltage is of the order of $\pm 0.01 \mu \mathrm{V}$. To allow measurement of these small signals, the laser radiation is chopped mechanically with a light chopper and a lock-in amplifier used to measure the voltage. The chopping frequency is nominally $400 \mathrm{~Hz}$. The frequency should be as high as possible while assuring the radiation stays on the specimen long enough during each cycle for the photovoltage to reach a steady-state value.

Motor Driven Stage - The specimen is held in a specimen holder (see next paragraph) which rests on a stepper-motor driven stage. The stepper motors are under control of the computer and are typically stepped so that measurements are made at $1-\mathrm{mm}$ intervals.

Specimen Holder-A picture of the specimen holder is shown in figure 5 . The holder is constructed so that measurement contact is made to the specimen on the top surface within about $\mathrm{l} \mathrm{mm}$ of the rim. Contact is made at four locations at 90-deg increments around the wafer (on perpendicular diameters) in a van der Pauw arrangement [6]. At each contact location, two probes (similar to those used for spreading resistance or four-probe measurements) make contact with the wafer and are separated by about $1.5 \mathrm{~mm}$.

The loading of each of the probes has been approximately 1.0 to $1.5 \mathrm{~N}$ in this work. During measurement of the bulk photovoltage, one of the probes at each end of the diameter being scanned is used to measure the voltage. During the measurement of the photoinduced change in specimen resistance, the other two probes (one at each end) are used to carry the constant current, and the change in potential across the specimen is measured with the same two probes as were used to measure the bulk photovoltage. Contact is made on perpendicular diameters to permit the measurement of the average specimen resistivity $(\bar{\rho})$ by the van der Pauw technique.

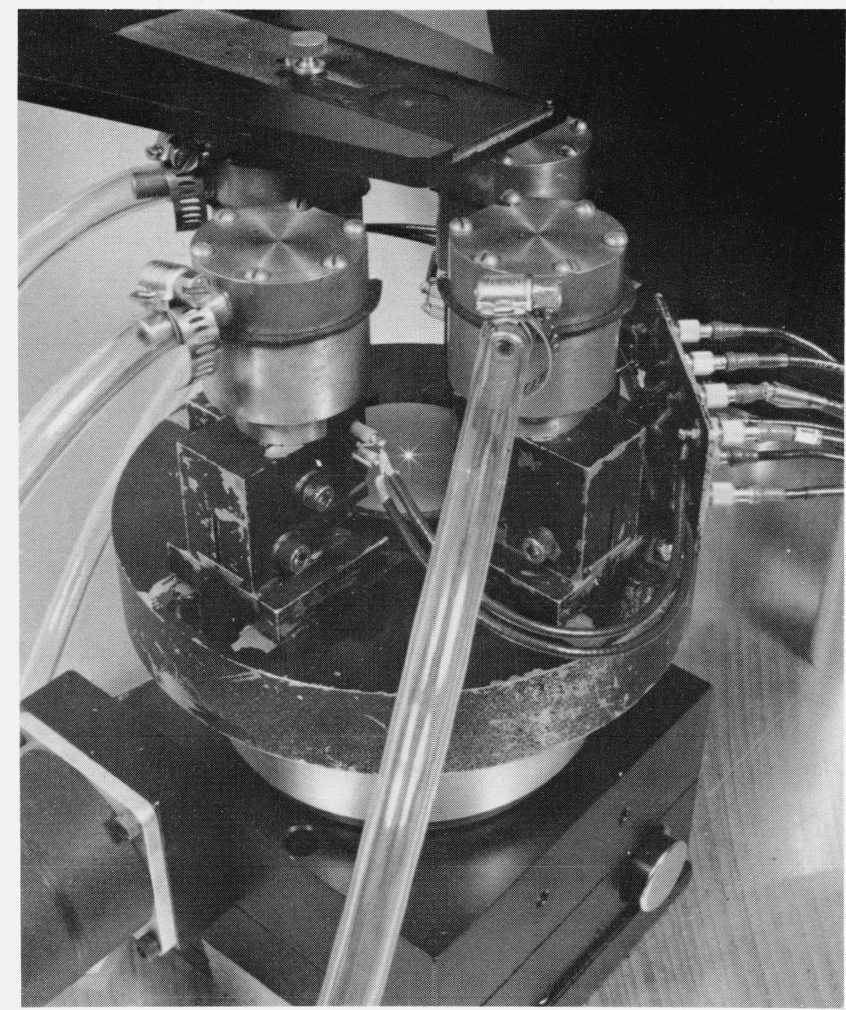

FIGURE 5. Picture of specimen holder.

\section{Results}

Two comparisons between the resistivity profiles as determined by the photovoltaic technique and by the four-probe method will be given to show the qualitative agreement that can be obtained. The comparisons are for high resistivity $(\sim 150 \Omega \cdot \mathrm{cm})$ and lower resistivity $(\sim 10 \Omega \cdot \mathrm{cm})$ specimens. The surfaces of the wafers were lapped with $12-\mu \mathrm{m}$ alumina. Measurements have been made on polished surfaces, but these measurements were not as reproducible as those made on lapped surfaces.

In figure 6 is shown a comparison between the respective resistivity profiles as determined for a nominal $150 \Omega \cdot \mathrm{cm}$, 


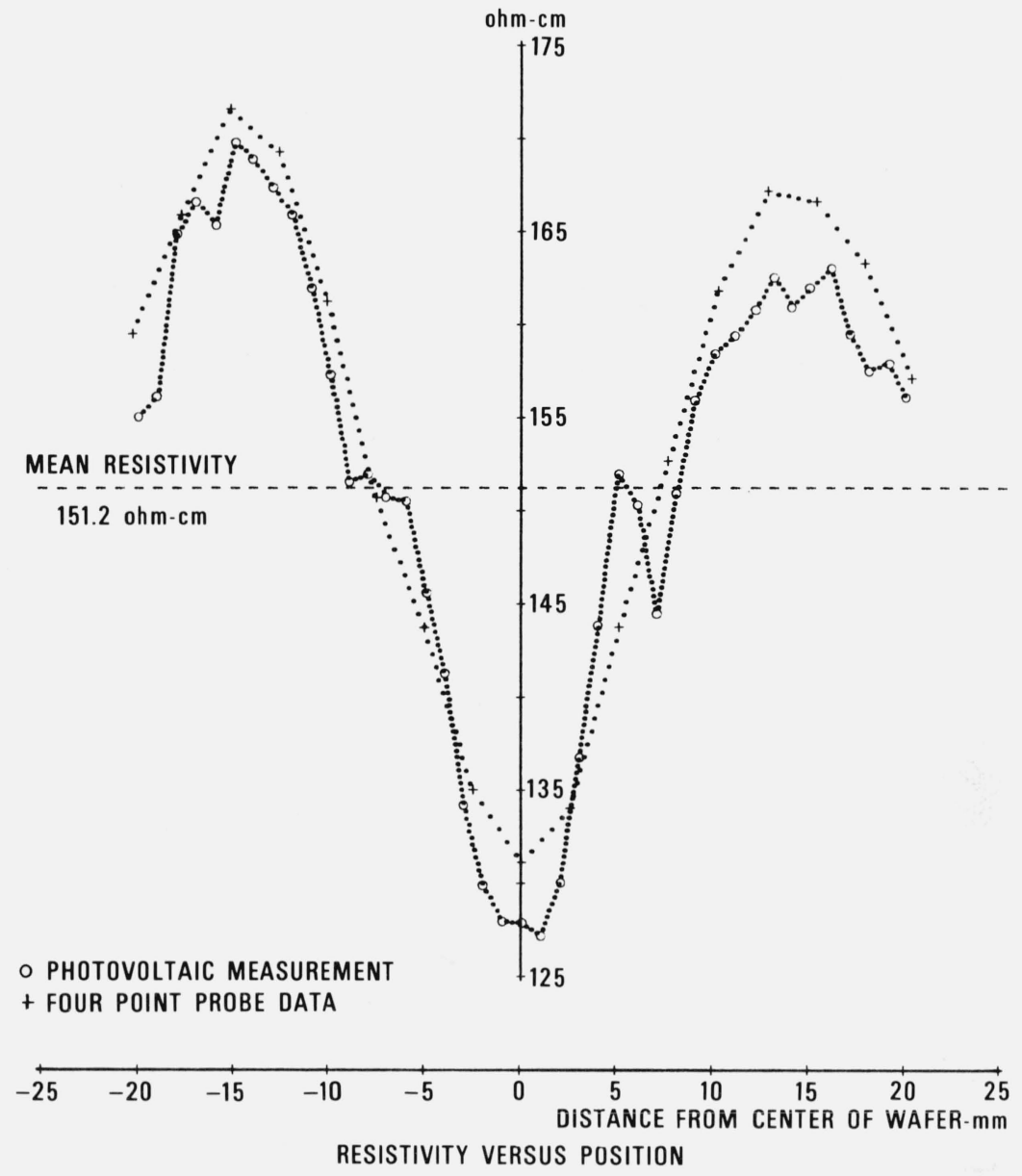

FiguRE 6. Comparison of resistivity profiles as determined by the four-probe technique and the photovoltaic technique for a nominal $150 \Omega \cdot \mathrm{cm}$-type silicon wafer.

$n$-type silicon wafer. The total time required to determine the photovoltaic resistivity profile on the 5 -cm diameter wafer was 2 to $3 \mathrm{~min}$. The total time required for measurement depends upon the magnitude of the photovoltage, which was between $-1 \mu \mathrm{V}$ to $1 \mu \mathrm{V}$ in this instance. The lock-in amplifier time constant was set to $100 \mathrm{~ms}$ and the light remained at each measurement point for about 20 time constants (i.e., $\sim 2 \mathrm{~s}$ ). Generally, for $\rho>50 \Omega \cdot \mathrm{cm}$, the 100 -ms time constant has been found satisfactory.

For lower resistivity specimens, the signal level is usually smaller because of a decrease in $d \rho / d x$, and also perhaps because of a decrease in minority carrier life-time. Thus, the lock-in amplifier time constant must be increased and the measurement time increased. A comparison of photovoltaic and four-probe resistivity profiles for a nominal $10 \Omega \cdot \mathrm{cm} n$ type silicon wafer is shown in figure 7 . The photovoltage for this specimen was usually in the range $-0.01 \mu \mathrm{V}$ to +0.01 $\mu \mathrm{V}$. A 300-ms lock-in amplifier time constant was used and the total measurement time was about $8 \mathrm{~min}$.

An indication of the reproducibility of the photovoltaic technique is given in figure 8. In this figure are shown the data for six resistivity profiles as determined by the photovoltaic technique along the same diameter of an $n$-type 135
$\Omega \cdot \mathrm{cm}$ silicon wafer. The measurements were made six different times over a period of several days. The wafer was removed and then reinserted into the specimen holder between measurements.

Detailed measurements to determine the spatial resolution of the photovoltaic technique have yet to be made. This will be done by comparing photovoltaic and spreading resistance resistivity profiles along the same wafer diameter. It is expected that the resolution will be one or two carrier diffusion lengths. In most high resistivity material, the diffusion length will be on the order of 0.5 to $1 \mathrm{~mm}$. Thus, a spatial resolution of no better than 1 to $2 \mathrm{~mm}$ may be expected, regardless of light probe size.

The minimum resistivity gradient which can be measured depends upon a number of parameters. The more electronhole pairs that are generated, the larger the signal for a given gradient and thus the smaller the minimum gradient measurable. Thus, the more intense the radiation source, the better the resistivity gradient resolution. Also, the longer the carrier lifetime, the better the resistivity gradient resolution (but the poorer the spatial resolution). In this work, the minimum gradient measurable has usually been of the order of 1.0 $\Omega \cdot \mathrm{cm} / \mathrm{cm}$ (a $0.1 \Omega \cdot \mathrm{cm}$ gradient over the $1-\mathrm{mm}$ diameter light probe). 


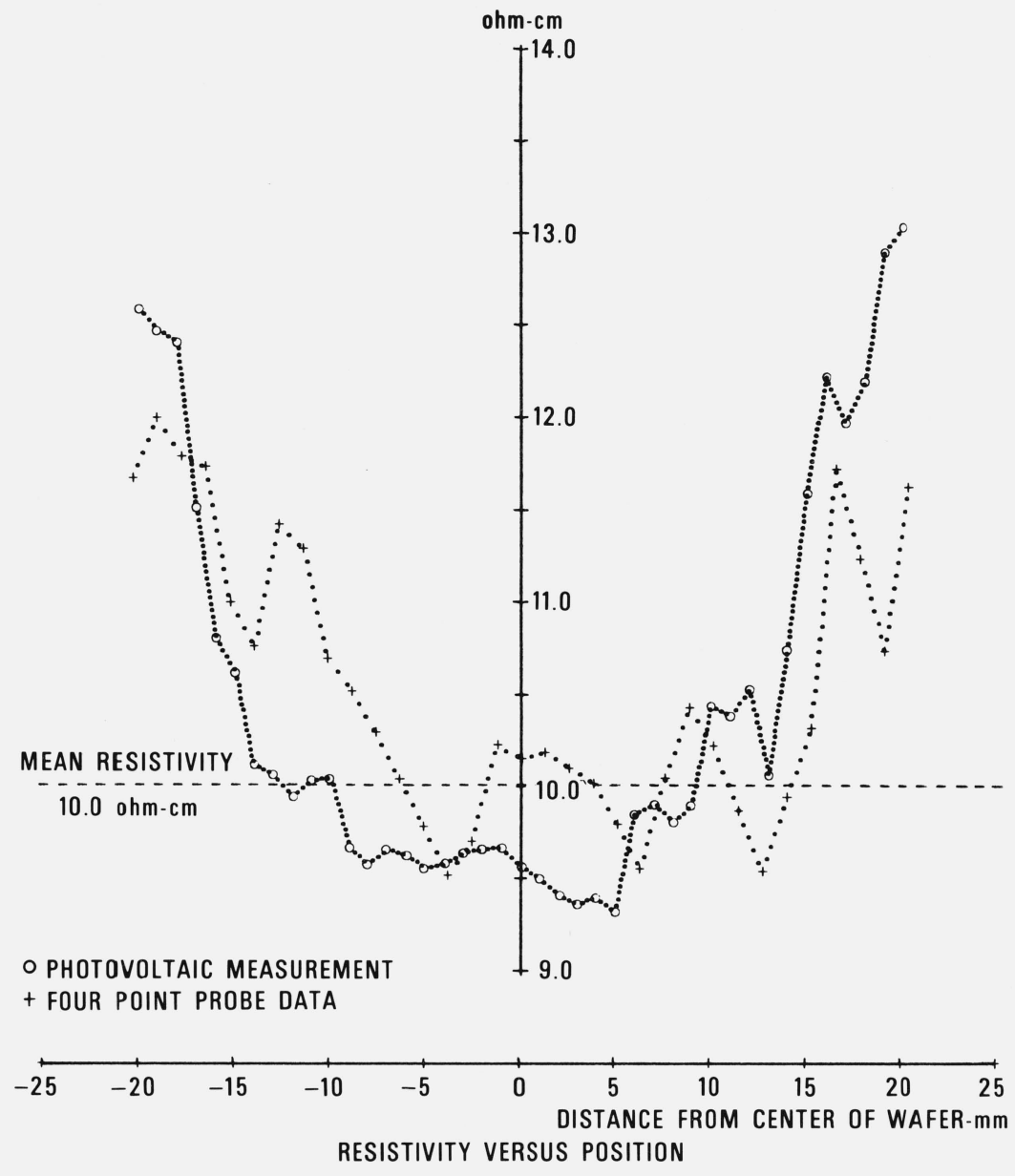

FIGURE 7. Comparison of resistivity profiles as determined by the four-probe technique and the photovoltaic technique for a nominal $10 \Omega \cdot \mathrm{cm}$-type silicon wafer.

Several photovoltaic resistivity measurements have been made on neutron transmutation doped (NTD) silicon. Because of the low resistivity gradients and the low lifetime usually present in this material, the bulk photovoltage has been extremely small; less than $0.01 \mu \mathrm{V}$. In addition, the presence of a front-to-back surface photovoltage interferes with the bulk photovoltage to such an extent that meaningful measurements on NTD material have not usually been possible. The front-to-back surface photovoltage is present on all specimens, but is only a problem when it is of the same order of magnitude as the bulk-photovoltage. This has been observed to be the case only in NTD silicon.

\section{Conclusion}

An automated system based upon the bulk photovoltaic effect for measuring the resistivity variations of high-resistiv- ity power grade silicon has been described. A brief description of the theory of the photovoltaic effect was given. Comparisons between the resistivity profiles as determined using the new system and as determined by the four-probe technique have been given as well as an indication of the reproducibility of the photovoltaic technique. In the work described, the measurement of variations of about $0.1 \Omega \cdot \mathrm{cm}$ for a l-mm light probe diameter was possible. The spatial resolution of the technique is expected to be about 1 to 2 minority carrier diffusion lengths. The time of measurements is about 2 to $3 \mathrm{~min}$ for $\rho \geq 50 \Omega \cdot \mathrm{cm}$ and 8 to $10 \mathrm{~min}$ for 10 $\leq \rho<50 \Omega \cdot \mathrm{cm}$.

The author gratefully acknowledges the contributions of R.D. Larrabee, G. J. Rogers and F. F. Oettinger to the results of this paper. 


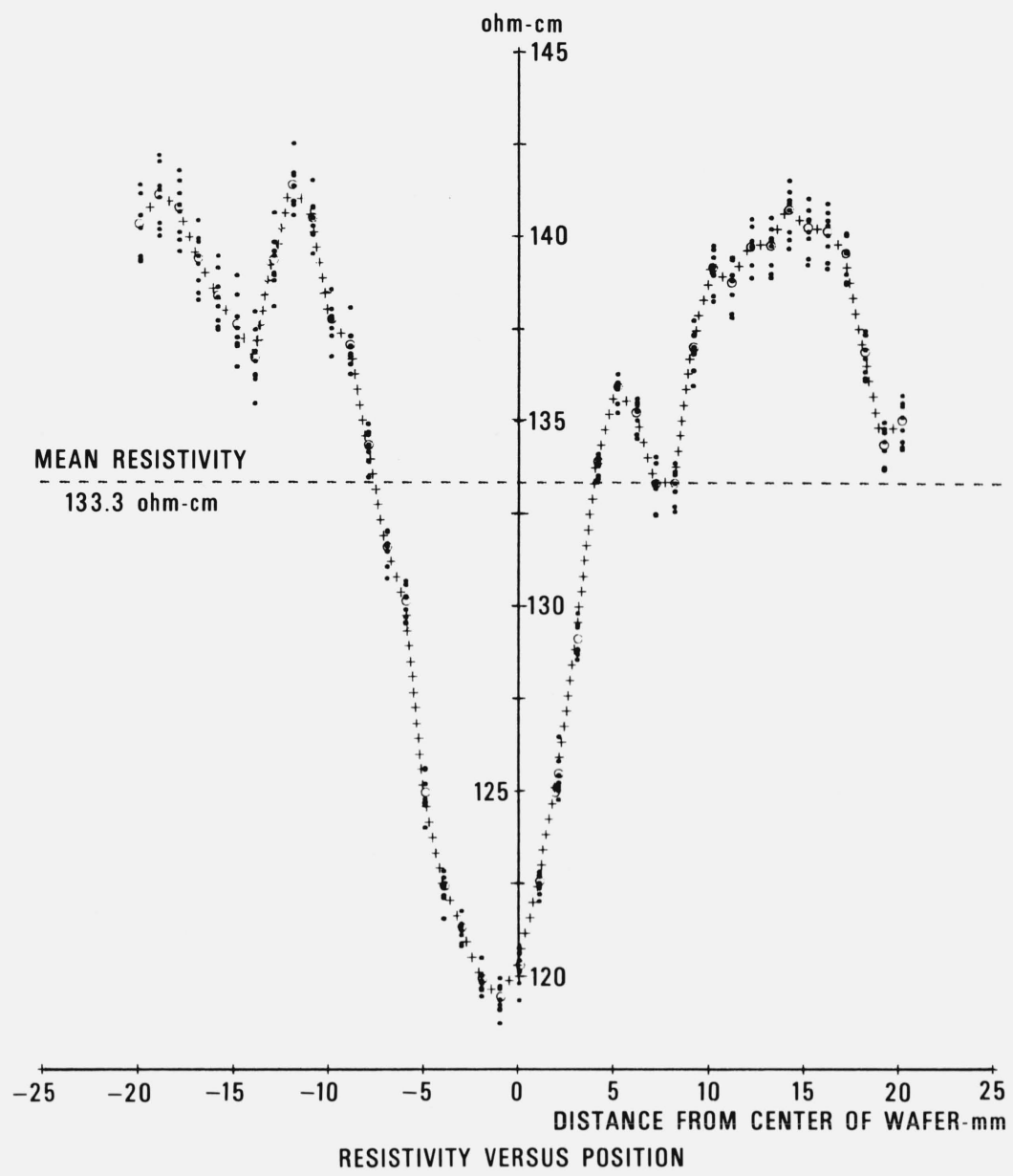

FIGURE 8. Repetitive photovoltaic resistivity profiles made along the same diameter of an ntype silicon wafer.

\section{References}

[1] Swartzendruber, L. J., Four-point probe measurement of non-uniformities in semiconductor sheet resistivity, Solid-State Electronics 7, 413-422 (1964).

[2] Vieweg-Gutberlet, F., and Schönhofer, F. X., Grensen der anwendbarkeit des 4-spitzen-gleichstrom-messverfahrens an silicium-proben, Part I, Arch. Tech. Messen. 369, 237-240 and Part II 370, 259262 (1966).

[3] Swartzendruber, L. J., Correction factor tables for four-point probe resistivity measurements on thin circular semiconductor samples, Natl. Bur. Std. (U.S.), Tech. Note 199, 34 pages (April 1964).

[4] Tauc, J., The Theory of a Bulk Photo-Voltaic Phenomenon in Semiconductors, Czech. J. Phys. 5, 178-179 (1955).

[5] Blackburn, D. L., Schafft, H. A., and Swartzendruber, L. J., Nondestructive Photovoltaic Techniques for the Measurement of Resistivity Gradients in Circular Semiconductor Wafers, J. Electrochem. Soc. 119, 1773-1778 (1972).

[6] van der Pauw, L. U., A Method of Measuring Specific Resistivity and Hall Effect of Discs of Arbitrary Shape, Philips Res. Rept. 13, 1-9 (1958). 